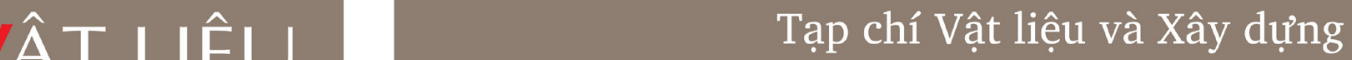 ISSN 1859 - 381X \\ Website: www.jomc.vn
}

\section{Xác định khả năng thắng thầu, giá dự thầu hợp lý trong đấu thầu cạnh tranh bằng phương pháp mạng Bayesian belief networks và lý thuyết trò chơi}

\author{
Nguyễn Đăng Trình ${ }^{1 *}$, Lê Thanh Tân ${ }^{2}$, Phan Duy Lai ${ }^{3}$, Trần Hoàng Gia ${ }^{1}$, Trần Đức Học ${ }^{1}$ \\ ${ }^{1}$ Khoa Kỹ Thuật Xây Dựng, Trường Đại Học Bách Khoa - Đại Học Quốc Gia Tp.HCM \\ ${ }^{2}$ Khoa Kiến trúc - Xây Dựng, Trường Đại học Bình Dương \\ ${ }^{3}$ Ban Quản lý dự án Mỹ Thuận \\ TỪ KHOÁ \\ Đấu thầu
}

Xây dựng

Lý thuyết trò chơi

Bayesian belief networks

\begin{abstract}
TÓM TẮT
Lựa chọn giá dự thầu hợp lý và xác định khả năng thắng thầu là một trong những vấn đề mang tính sống còn đối với các nhà thầu. Đây là một vấn đề rất khó vì trong hoạt động đấu thầu có sự đa dạng và phức tạp. Các nghiên cứu trước chỉ dựa vào dữ liệu quá khứ để đưa ra quyết định; tuy nhiên, dữ liệu quá khứ chỉ là một nhân tố ảnh hưởng đến quyết định tham gia dự thầu cần thêm các dữ liệu thông tin tổng hợp khác. Bài báo này trình bày phương pháp sử dụng mạng bayesian belief networks (BBNs) và lý thuyết trò chơi để xác định khả năng thắng thầu trong đấu thầu cạnh tranh. Để thực hiện các mục tiêu trên, Kết quả nghiên cứu đã tiến hành thu thập dữ liệu và phân tích, để đưa ra các nhân tố ảnh hưởng có thể đánh giá được bản thân và các đối thủ sẽ có xu hướng lựa chọn giá dự thầu. Đồng thời, mô hình cũng đánh giá sự tương tác của nhà thầu với các đối thủ tiềm năng để xác định giá dự thầu hợp lý, cũng như xác suất thắng thầu lớn nhất.
\end{abstract}

\section{KEYWORDS}

Bidding

Construction

Game theory

Bayesian belief networks

\begin{abstract}
Selecting a reasonable bidding price and determining the ability to win the bidding process are vital issues for contractors. It is a very difficult problem because there are diversity and complexity in bidding activities. Previous studies only relied on historical data to make decisions; however, the historical data is only one factor affecting the decision to participate in the bidding process. This study uses bayesian belief networks (BBNs) and game theory to determine the likelihood of winning a bid in competitive bidding. To achieve the above objectives, research has conducted to collect data and analyze to identify influencing factors that can be assessed for contractors and competitors will be more inclined to choose bid prices. At the same time, the model also assesses the bidder's interaction with potential competitors to determine a reasonable bid price, as well as the greatest probability of winning.
\end{abstract}

\section{Giới thiệu, tổng quan mô hình}

Mô hình xác định giá dự thầu hợp lý, xác suất thắng thầu được tác giả đề xuất thực hiện qua bốn bước sau:

Bước 1: Xác định các nhân tố chính ảnh hưởng đến quyết định. Xác định khoảng, mức độ xảy ra của nhân tố, giá trị lợi nhuận (mark-up) và chiến thuật giá tương ứng để xây dựng mối quan hệ.

Bước 2: Xây dựng mối quan hệ các mức độ xảy ra các nhân tố và xu hướng lựa chọn các chiến thuật giá.

Bước 3: Xây dựng mạng BBNs mối quan hệ nguyên nhân - kết quả, các mối tương quan giữa các nhân tố, để xác định xác suất lựa chọn các chiến thuật (sau khi có dữ liệu đầu vào là phân bố xác suất các mức độ).
Bước 4: Sử dụng lý thuyết trò chơi để đánh giá sự tương tác giữa các bên tham gia (sau khi có được dữ liệu đầu vào là phân bố xác suất các trường hợp tương quan giá gốc của các bên tham gia).

2. Phân chia các mức độ các nhân tố ảnh hưởng, khảo sát xác định sự ảnh hưởng đến xu hướng lựa chọn các chiến thuật giá

Trong giới hạn của nghiên cứu, tác giả tổng hợp và lập một danh mục nhân tố gồm 28 nhân tố chính, được chia thành bốn nhóm nhân tố chính, ảnh hưởng đến quyết định lựa chọn giá trị mark-up từ các nghiên cứu trước (Hình 2) [1], [2], [3], [4].

Các chiến thuật sẽ được chia ra nhiều mức độ xảy ra theo thứ bậc từ nhiều đến ít, từ lớn đến nhỏ. Sau đó, tác giả sẽ tiến hành khảo sát xu hướng lựa chọn các chiến thuật giá (Hình 3).

\footnotetext{
"Tác giả liên hệ: ndtrinh@hcmut.edu.vn

Nhận ngày 02/04/2021, giải trình ngày 15/05/2021, chấp nhận đăng 18/07/2021
} 


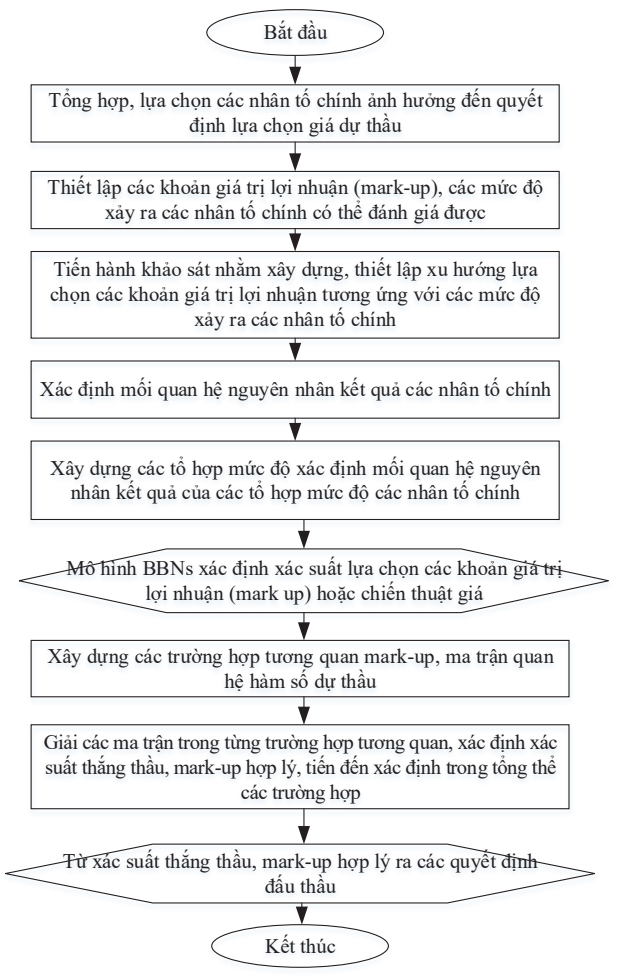

Hình 1. Phương pháp xác định khả năng thắng thầu, giá dự thầu hợp lý.

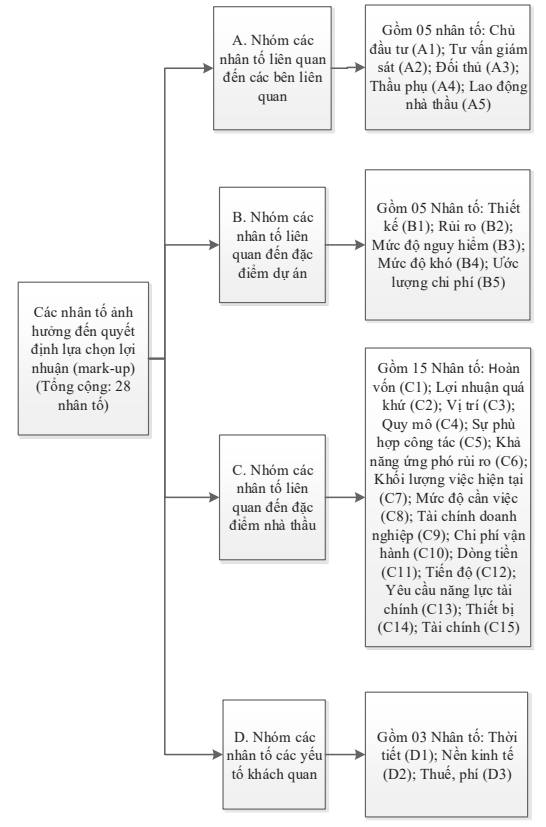

Hình 2. Các nhân tố ảnh hưởng đến quyết định lựa chọn lợi nhuận.

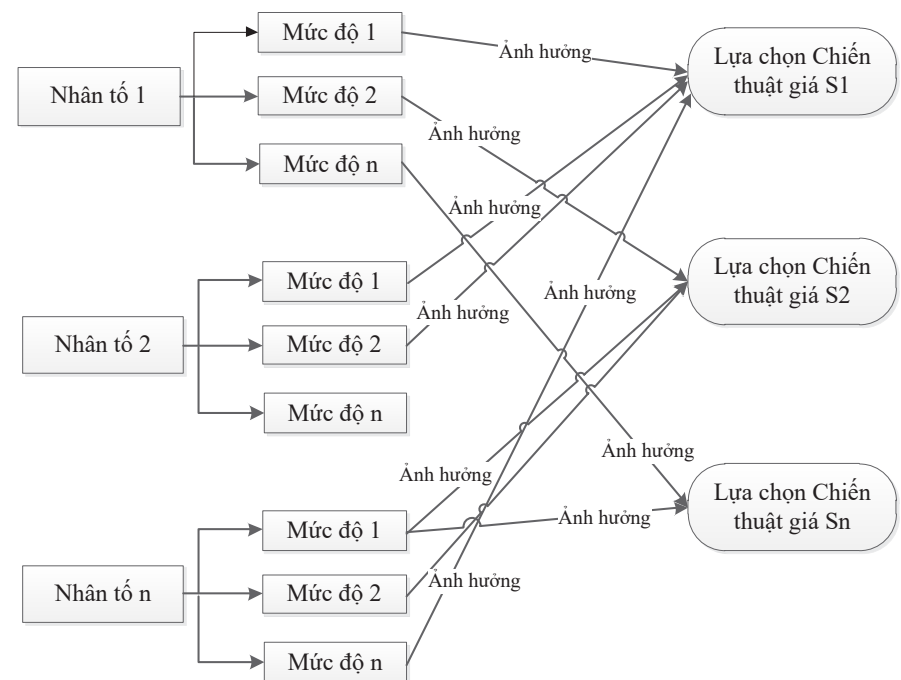

Hình 3. Minh họa cách thức lựa chọn chiến thuật giá khi các nhân tố xảy ra ở các mức độ khác nhau.

Trong giới hạn của nghiên cứu, mỗi nhân tố được tác giả chia làm ba mức độ (theo thứ bậc từ cao đến thấp, từ nhiều đến ít), để tiến hành khảo sát xu hướng lựa chọn ba chiến thuật giá. Phương pháp TTest, Cronbach's alpha được dùng để kiểm định kết quả khảo sát, mà các đối tượng được khảo sát chủ yếu đã từng hoặc đang công tác trong các đơn vị thi công xây lắp, và có các vị trí từ lãnh đạo đến nhân viên (chủ yếu liên quan đến công tác đấu thầu). Kết quả của quá trình này sẽ giúp xác định được với mỗi mức độ lợi nhuận sẽ là tổ hợp các nhân tố, với các mức độ xảy ra tương ứng.

\section{Vận dụng mạng BBNs để xác định xác suất lựa chọn các chiến thuật giá dự thầu}

Từ những ý kiến của chuyên gia và các nghiên cứu trước, tác giả tiến hành lập bảng mối quan hệ nguyên nhân - kết quả của các nhân tố ảnh hưởng. Sau đó, tác giả gửi bảng mối quan hệ này đến các đối tượng được khảo sát (thành phần được khảo sát tương tự như mục 2).

Kết quả khảo sát được tác giả thực hiện kiểm định T-Test, Cronbach's alpha. Đây là cơ sở để tác giả xây dựng mạng BBNs. Vấn đề chủ yếu của việc xây dựng mạng BBNs là xác định xác suất trạng thái của 02 biến lớn là biến trung gian, biến nhân tố kết quả.

\section{1. Đối với biến trung gian}

Trạng thái biến: Khả năng xác suất nhà thầu chọn chiến thuật Si (Yes); khả năng xác suất nhà thầu không chọn chiến thuật Si (No) 
Gọi $(1-x)$ là mức độ $\mathrm{x}$ của nhân tố 1 , với $x=\{1,2,3\}$, được sắp xếp theo thứ tự từ cao đến thấp, từ lớn đến nhỏ. Các tổ hợp có thể tồn tại được minh họa trong Bảng 1.

Bảng 1.

Các tổ hợp nhân tố - mức độ ảnh hưởng đến trạng thái biến trung gian.

\begin{tabular}{|c|c|c|c|c|}
\hline & & $k-1$ & $k-2$ & $\begin{array}{l}k-3 \\
\left(\mathrm{~S}_{1}\right) \\
\end{array}$ \\
\hline \multirow{4}{*}{$\begin{array}{l}l-1 \\
\left(\mathrm{~S}_{1}\right)\end{array}$} & $m-1$ & $\begin{array}{l}\text { Tổ hợp } 1 \\
\text { (Hỗn hợp) }\end{array}$ & $\begin{array}{l}\text { Tổ hợp } 2 \\
\text { (Hỗn hợp) }\end{array}$ & $\begin{array}{l}\text { Tổ hợp } 3 \\
\text { (Hỗn hợp) }\end{array}$ \\
\hline & $m-2$ & Tổ hợp 4 & Tổ hợp 5 & Tổ hợp 6 \\
\hline & $\left(S_{1}\right)$ & (Hỗn hợp) & (Hỗn hợp) & Yes $=1$ \\
\hline & $m-3$ & $\begin{array}{l}\text { Tổ hợp } 7 \\
\text { (Hỗn hợp) }\end{array}$ & $\begin{array}{l}\text { Tổ hợp } 8 \\
\text { (Hỗn hợp) }\end{array}$ & $\begin{array}{l}\text { Tổ hợp } 9 \\
\text { (Hỗn hợp) }\end{array}$ \\
\hline \multirow{6}{*}{$l-2$} & & Tổ hợp 10 & Tổ hợp 11 & Tổ hợp 12 \\
\hline & $m-1$ & $\mathrm{No}=1$ & No $=1$ & (Hỗn hợp) \\
\hline & & Tổ hợp 13 & Tổ hợp 14 & Tổ hợp 15 \\
\hline & $m-2$ & $\mathrm{No}=1$ & No $=1$ & (Hỗn hợp) \\
\hline & & Tổ hợp 16 & Tổ hợp 17 & Tổ hợp 18 \\
\hline & $m-3$ & No $=1$ & No $=1$ & (Hỗn hợp) \\
\hline \multirow{6}{*}{$l-3$} & & Tổ hợp 19 & Tổ hợp 20 & Tổ hợp 21 \\
\hline & $m-1$ & $\mathrm{No}=1$ & $\mathrm{No}=1$ & (Hỗn hợp) \\
\hline & & Tổ hợp 22 & Tổ hợp 23 & Tổ hợp 24 \\
\hline & $m-2$ & $\mathrm{No}=1$ & $\mathrm{No}=1$ & (Hỗn hợp) \\
\hline & & Tổ hợp 25 & Tổ hợp 26 & Tổ hợp 27 \\
\hline & $m-3$ & No $=1$ & No $=1$ & (Hỗn hợp) \\
\hline
\end{tabular}

Giả sử kết quả khảo sát chỉ ra rằng xu hướng lựa chọn ba chiến thuật $S_{1}$ các nhân tố $l, m, k$ như sau:

$$
S_{1}=\{(l-1) ;(m-2) ;(k-3)\}
$$

Biến trung gian gồm 02 trạng thái (Yes, No), tương ứng với xác suất $P\left(S_{1}\right.$ Yes $) ; P\left(S_{1} N o\right)$.

Xác suất của các tổ hợp xu hướng mức độ chắc chắn lựa chọn chiến thuật có dạng $P(Y e s \mid(l-1),(m-2),(k-3))=1$ - Tổ hợp số 6, việc này đồng nghĩa với $P(N o \mid(l-1),(m-2),(k-3))=0$.

Xác suất của các tổ hợp có xu hướng mức độ chắc chắc không lựa chọn $P\left(S_{1} N o\right)$ là tổ hợp các mức độ nhân tố không thuộc $S_{1}=\{(l-1) ;(m-2) ;(k-3)\}$, được trình bày trong Bảng 1 , gồm các tổ hợp số $10,11,13,14,16,17,19,20,22,23,25$ và 26.

Ví dụ: Tổ hợp số 10 có $P(N o \mid(l-2),(m-1),(k-1))=1$, đồng nghĩa với $P(\operatorname{Yes} \mid(l-2),(m-1),(k-1))=0$.

Các tổ hợp tồn tại một số thành phần mức độ nhân tố thuộc $S_{1}=\{(l-1) ;(m-1) ;(k-1)\}$, tạm gọi là tổ hợp hỗn hợp, có xác suất chắc chắn chọn tùy thuộc vào hệ số $\alpha=\{0 ; 1\}$ (là mức độ tin tưởng mức độ nhân tố thuộc $S_{1}$ tham gia tổ hợp), được trình bày trong Bảng 1 , gồm các tổ hợp số $1,2,3,4,5,7,8,9,12,15,18,21,24$ và 27.

Ví dụ: Tổ hợp số 1 có hệ số tin tưởng $(l-1) \in S_{1}$ là $\alpha$. Ta sẽ có $P($ Yes $\mid(l-1),(m-1),(k-1))=\alpha, \alpha=\{0,1\} \quad, \quad$ đồng nghĩa với $P(N o \mid(l-1),(m-1),(k-1))=1-\alpha$.

\section{2. Đối với biến kết quả}

Trạng thái biến: ba mức độ xảy ra đối với nhân tố kết quả a là $(\mathrm{a}-1),(\mathrm{a}-2),(\mathrm{a}-3)$.

Các nhân tố nguyên nhân - kết quả tồn tại mối quan hệ tương quan được xác định qua hệ số quan hệ K, gồm có quan hệ thuận chiều và quan hệ trái chiều được minh họa trong Hình 4.

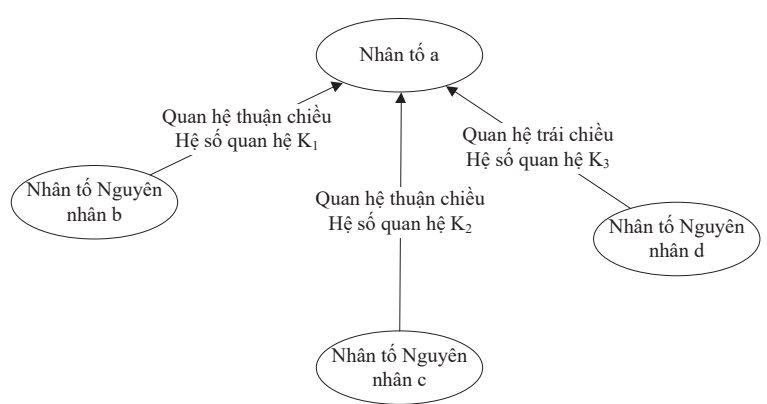

Hình 4. Minh họa mối quan hệ nguyên nhân - kết quả giữa các nhân tố.

Giả sử kết quả sau khi khảo sát thu được: Nhân tố a là kết quả của các nhân tố nguyên nhân, gồm Nhân tố b, Nhân tố c và Nhân tố $\mathrm{d}$. Trong đó, mức độ quan hệ được xác định như sau:

- Nhân tố a và Nhân tố b quan hệ thuận chiều tỷ lệ $\mathrm{K}_{1}$;

- Nhân tố a và Nhân tố c quan hệ nghịch chiều tỷ lệ $\mathrm{K}_{2}$;

- Nhân tố a và Nhân tố d quan hệ nghịch chiều tỷ lệ $\mathrm{K}_{3}$;

- Nhân tố a có các trạng thái tương ứng với ba mức độ xảy ra là (a-1), (a-2) và (a-3).

\section{Bảng 2.}

Các tổ hợp nhân tố nguyên nhân ảnh hưởng đến trạng thái biến nhân tố kết quả.

\begin{tabular}{|c|c|c|c|c|}
\hline & & d-1 & $\mathrm{d}-2$ & d-3 \\
\hline \multirow{3}{*}{ b-1 } & $c-1$ & $\begin{array}{l}\text { Tổ hợp } 1 \\
\text { (Hỗn hợp) }\end{array}$ & $\begin{array}{l}\text { Tổ hợp } 2 \\
\text { (a-3) =0 }\end{array}$ & $\begin{array}{l}\text { Tổ hợp } 3 \\
(a-2)=0 \\
(a-3)=0\end{array}$ \\
\hline & $\mathrm{c}-2$ & $\begin{array}{l}\text { Tổ hợp } 4 \\
\text { (Hỗn hợp) }\end{array}$ & $\begin{array}{l}\text { Tổ hợp } 5 \\
(\mathrm{a}-3)=0\end{array}$ & $\begin{array}{l}\text { Tổ hợp } 6 \\
(\mathrm{a}-3)=0\end{array}$ \\
\hline & $c-3$ & $\begin{array}{l}\text { Tổ hợp } 7 \\
\text { (Hỗn hợp) }\end{array}$ & $\begin{array}{l}\text { Tổ hợp } 8 \\
\text { (Hỗn hợp) }\end{array}$ & $\begin{array}{l}\text { Tổ hợp } 9 \\
\text { (Hỗn hợp) }\end{array}$ \\
\hline
\end{tabular}




\begin{tabular}{|c|c|c|c|c|}
\hline & & $\mathrm{d}-1$ & $\mathrm{~d}-2$ & d-3 \\
\hline \multirow{3}{*}{ b-2 } & c-1 & $\begin{array}{l}\text { Tổ hợp } 10 \\
\text { (Hỗn hợp) }\end{array}$ & $\begin{array}{c}\text { Tổ hợp } 11 \\
(\mathrm{a}-3)=0\end{array}$ & $\begin{array}{c}\text { Tổ hợp } 12 \\
(\mathrm{a}-3)=0\end{array}$ \\
\hline & $\mathrm{c}-2$ & $\begin{array}{c}\text { Tổ hợp } 13 \\
\text { (a-1)=0 }\end{array}$ & $\begin{array}{c}\text { Tổ hợp } 14 \\
\begin{array}{c}(\mathrm{a}-1)=0 \\
(\mathrm{a}-3)=0\end{array}\end{array}$ & $\begin{array}{c}\text { Tổ hợp } 15 \\
\text { (a-3) }=0\end{array}$ \\
\hline & $c-3$ & $\begin{array}{c}\text { Tổ hợp } 16 \\
(\mathrm{a}-1)=0\end{array}$ & $\begin{array}{c}\text { Tổ hợp } 17 \\
(\mathrm{a}-1)=0\end{array}$ & $\begin{array}{l}\text { Tổ hợp } 18 \\
\text { (Hô̂n hợp) }\end{array}$ \\
\hline \multirow{3}{*}{ b-3 } & c-1 & $\begin{array}{l}\text { Tổ hợp } 19 \\
\text { (Hỗn hợp) }\end{array}$ & $\begin{array}{l}\text { Tổ hợp } 20 \\
\text { (Hỗn hợp) }\end{array}$ & $\begin{array}{l}\text { Tổ hợp } 21 \\
\text { (Hỗn hợp) }\end{array}$ \\
\hline & $\mathrm{c}-2$ & $\begin{array}{c}\text { Tổ hợp } 22 \\
(\mathrm{a}-1)=0\end{array}$ & $\begin{array}{c}\text { Tổ hợp } 23 \\
(\mathrm{a}-1)=0\end{array}$ & $\begin{array}{l}\text { Tổ hợp } 24 \\
\text { (Hỗn hợp) }\end{array}$ \\
\hline & c-3 & $\begin{array}{c}\text { Tồ hợp } 25 \\
(\mathrm{a}-2)=0 \\
(\mathrm{a}-1)=0\end{array}$ & $\begin{array}{c}\text { Tổ hợp } 26 \\
\text { (a-1) }=0\end{array}$ & $\begin{array}{l}\text { Tổ hợp } 27 \\
\text { (Hô̂n hợp) }\end{array}$ \\
\hline
\end{tabular}

Khả năng xảy ra hoàn toàn với mức độ $i=\{1,2,3\}$ của nhân tố a sẽ là kết quả của khả năng xảy ra nhân tố $b, c$ (quan hệ thuận chiều) và nhân cố d (quan hệ ngược chiều) với các mức độ tương ứng, được trình bày trong Bảng 2 .

Nhóm các tổ hợp có hai mức độ khả năng không xảy ra, gồm tổ hợp số 3,14 và 25 . Ví dụ: Tổ hợp số 3 với khả năng xảy ra hai mức độ $(\mathrm{a}-2)$ và $(\mathrm{a}-3)=0$ thì khả năng xảy ra (a-1) sẽ là $P((a-1) \mid(b-1),(c-1),(d-3))=K_{1} \times K_{2} \times K_{3}$.

Trường hợp có từ 02 mức độ có khả năng xảy ra, việc xem xét tương quan, ngoài việc xem xét tỷ lệ các mối quan hệ, để đánh giá tốt các tổ hợp hỗn hợp có ba mức độ có thể xảy ra và có hai mức độ có thể xảy ra, thì việc xem xét này nên được thực hiện trong từng tổ hợp. Bởi vì thực tế mối quan hệ trong đấu thầu là một vấn đề phức tạp, phụ thuộc vào nhiều vấn đề khác. Ta phải xác định được các hệ số các mối quan hệ mức độ $\beta$ để xác định xác suất trong các trường hợp. Ví dụ theo dữ liệu của Bảng 3, tổ hợp mối quan hệ giữa mức độ (b-1) nhân tố b với các mức độ (a-1) và (a-2) là $\beta_{(a-1)}^{(b-1)}=1, \beta_{(a-2)}^{(b-1)}=0, \beta_{(a-1)}^{(b-1)}+\beta_{(a-2)}^{(b-1)}=1$.

\section{Bảng 3.}

Đánh giá sự ảnh hưởng các mức độ trong Tổ hợp 2 (các mức độ nhân tố nguyên nhân) đến 02 mức độ có khả năng xảy ra của nhân tố kết quả a.

\begin{tabular}{cccc}
\hline & & \multicolumn{2}{c}{ Nhân tố kết quả a } \\
\cline { 2 - 4 } & & $\mathrm{a}-1$ & $\mathrm{a}-2$ \\
\hline & $\mathrm{b}-1$ & 1 & 0 \\
Tổ hợp 2 & $\mathrm{c}-1$ & 0,7 & 0,3 \\
& $\mathrm{~d}-2$ & 0,2 & 0,8 \\
\hline & $\mathrm{TC}$ & 1,9 & 1,1 \\
\hline & $\beta$ & 0,63 & 0,36 \\
\hline
\end{tabular}

Bảng 4.

Đánh giá sự ảnh hưởng các mức độ trong Tổ hợp 1 (các mức độ nhân tố nguyên nhân) đến 03 mức độ có khả năng xảy ra của nhân tố kết quả a.

\begin{tabular}{ccccc}
\hline & & \multicolumn{4}{c}{ Nhân tố kết quả a } \\
\cline { 3 - 5 } & & $\mathrm{a}-1$ & $\mathrm{a}-2$ & $\mathrm{a}-3$ \\
\hline & $\mathrm{b}-1$ & 1 & 0 & 0 \\
Tổ hợp 1 & $\mathrm{c}-1$ & 0,7 & 0,2 & 0,1 \\
& $\mathrm{~d}-1$ & 0,05 & 0,1 & 0,85 \\
& TC & 1,75 & 0,3 & 0,95 \\
\hline & $\beta$ & 0,58 & 0,1 & 0,32 \\
\hline
\end{tabular}

Nhóm các tổ hợp có một mức độ khả năng không xảy ra, gồm tổ hợp số $2,5,6,11,12,13,15,16,17,22,23$ và 26 (Bảng 2). Ví dụ tại tổ hợp số 2 , khả năng xảy ra mức độ $(\mathrm{a}-3)=0$ thì khả năng xảy ra sẽ được chia cho hai mức độ còn lại là (a-1) và (a-2), được xác định thông qua hệ số thể hiện mức độ tin tưởng khả năng xảy ra $\beta=\{0,1\}$ $P((a-1) \mid(b-1),(c-1),(d-1))=K_{1} \times K_{2} \times K_{3} \times \beta$, đối với mức độ (a-2) là: $P((a-1) \mid(b-1),(c-1),(d-3))=K_{1} \times K_{2} \times K_{3} \times(1-\beta)$.

Theo Bảng 3 , khả năng xảy ra mức độ (a-1) của nhân tố a là $P((a-1) \mid(b-1),(c-1),(d-1))=K_{1} \times K_{2} \times K_{3} \times 0,63$, và với mức độ (a2) là $P((a-1) \mid(b-1),(c-1),(d-1))=K_{1} \times K_{2} \times K_{3} \times 0,36$.

Nhóm các tổ hợp có ba mức độ có khả năng xảy ra (tổ hợp hỗn hợp), gồm tổ hợp số $1,4,7,8,9,10,18,19,20,21,24$ và 27 . Ví dụ tổ hợp số 1 (Bảng 4), khả năng xảy ra mức độ (a-3) $=0$ thì khả năng xảy ra sẽ được chia cho hai mức độ còn lại là (a-1) và (a-2).

Bảng 4 minh họa khả năng xảy ra mức độ (a-1) của nhân tố a là $P((a-1) \mid(b-1),(c-1),(d-1))=K_{1} \times K_{2} \times K_{3} \times 0,58$, khả năng xảy ra với mức độ (a-2) là $P((a-1) \mid(b-1),(c-1),(d-1))=K_{1} \times K_{2} \times K_{3} \times 0,1$, và với mức độ (a-3) là $P((a-1) \mid(b-1),(c-1),(d-1))=K_{1} \times K_{2} \times K_{3} \times 0,32$

\section{Vận dụng Lý thuyết trò chơi để đánh giá sự tương tác}

Gọi $C^{i}$ là chi phí nhà thầu $i$ phải bỏ ra để thực hiện gói thầu theo yêu cầu về chất lượng mà bên mời thầu yêu cầu. Đây là giá cơ bản (hay giá gốc) của nhà thầu.

Gọi $B_{\alpha}^{i}$ là giá dự thầu của nhà thầu i tại chiến thuật thứ $\alpha, \forall \alpha \in$ $n=\{1,2, \ldots, N\}$.

Giả sử giá trị lợi nhuận cho từng chiến thuật của các nhà thầu là như nhau $B_{\alpha+1}^{i}-B_{\alpha}^{i}=\Delta$. Cụ thể các phương án lợi nhuận như sau:

Với nhà thầu i: $n \Delta, n=\{1,2, \ldots, N\}$ tương ứng với $\mathrm{n}$ chiến thuật lợi nhuận $S_{n}^{i}$, 


$$
\begin{aligned}
& S_{1}^{i}: B_{1}^{i}=C^{i}+\Delta \\
& S_{2}^{i}: B_{2}^{i}=C^{i}+2 \Delta \\
& S_{n}^{i}: B_{n}^{i}=C^{i}+n \Delta
\end{aligned}
$$

Với nhà thầu $\mathrm{j}: n \Delta, n=\{1,2, \ldots, N\}$ tương ứng với $\mathrm{n}$ chiến thuật lợi nhuận $S_{n}^{j}$,

$$
\begin{aligned}
& S_{1}^{j}: B_{1}^{j}=C^{j}+\Delta \\
& S_{2}^{j}: B_{2}^{j}=C^{j}+2 \Delta \\
& S_{n}^{j}: B_{n}^{j}=C^{j}+n \Delta
\end{aligned}
$$

Gọi $S_{\alpha}^{i}, S_{\beta}^{j}$ là chiến thuật của hai nhà thầu $i$ và $j$. Trường hợp nhà thầu $i$ chiến thắng nhà thầu $j\left(B_{\alpha}^{i}<B_{\beta}^{j}\right)$ thì hàm số dự thầu sẽ có dạng như sau $U_{\alpha}^{i}=B_{\alpha}^{i}-C^{i}$. Ngược lại, nhà thầu $j: U_{\beta}^{j}=0$ Các nhà thầu sẽ phải tối đa hóa lợi nhuận, nhưng vẫn phải đảm bảo cho việc trúng thầu. Giá dự thầu hợp lý của nhà thầu $i$ sẽ thỏa công thức sau [5], [6]:

$$
\mathrm{UP}_{n}^{i}=\operatorname{Max}\left\{\left(B_{\alpha}^{i}-C^{i}\right) \cdot \operatorname{prob}\left(B_{\alpha}^{i}<B_{\beta}^{j}\right)+\frac{B_{\alpha}^{i}-C^{i}}{2} \cdot \operatorname{prob}\left(B_{\alpha}^{i}=B_{\beta}^{j}\right)+0\right\}
$$

Xác suất xảy ra giá dự thầu hai nhà thầu bằng nhau là rất bé, ta bỏ qua trường hợp này: $\operatorname{prob}\left(B_{\alpha}^{i}=B_{\beta}^{j}\right)=0$.

$$
\begin{aligned}
& \mathrm{UP}_{n}^{i}=\operatorname{Max}\left\{\left(B_{\alpha}^{i}-C^{i}\right) \cdot \operatorname{prob}\left(B_{\alpha}^{i}<B_{\beta}^{j}\right)+\frac{B_{\alpha}^{i}-C^{i}}{2} \cdot \operatorname{prob}\left(B_{\alpha}^{i}=B_{\beta}^{j}\right)+0\right\} \\
& =\operatorname{Max}\left\{\left(B_{\alpha}^{i}-C^{i}\right) \cdot \operatorname{prob}\left(B_{\alpha}^{i}<B_{\beta}^{j}\right)\right\}
\end{aligned}
$$

Theo Dong-hong và các đồng sự [5], đây là chiến thuật hợp lý của các nhà thầu tham gia.

Như đã trình bày ở mục 1 , dữ liệu đầu vào của việc đánh giá tương tác là phân bố xác suất tương quan giá gốc của các bên tham gia: $\operatorname{prob}\left(C^{i}<C^{j}\right), \operatorname{prob}\left(C^{i}>C^{j}\right)$, và việc cả hai nhà thầu có mức giá cơ bản bằng nhau rất khó xảy $\operatorname{ra} \operatorname{prob}\left(C_{i}=C_{j}\right)=0$ với: $\operatorname{prob}\left(C^{i}<C^{j}\right)+$ $\operatorname{prob}\left(C^{i}>C^{j}\right)=1$.

Với $\operatorname{prob}\left(C^{i}<C^{j}\right)$ : Có $\mathrm{n}$ trường hợp tương quan giá dự thầu $C^{i}+$ $(n+1) \Delta<C^{j}, n=1,2, \ldots, N$ tương ứng với các mức xác suất $\operatorname{prob}[(n-1) \Delta]$. Nhà thầu sẽ có ma trận $n \times n$ tình huống. Trong giới hạn nghiên cứu, tác giả giả sử mỗi nhà thầu sẽ sử dụng ba phương án chiến thuật, từ đó, ma trận $3 \times 3$ được lập như Bảng 5 .

\section{Bảng 5.}

Ma trận tình huống nhà thầu i, j.

\begin{tabular}{ccccc}
\hline & \multicolumn{4}{c}{ Nhà thầu i } \\
\hline & & $S_{1}^{i}$ & $S_{2}^{i}$ & $S_{3}^{i}$ \\
Nhà & $S_{1}^{j}$ & $U_{11}^{i} ; U_{11}^{j}$ & $U_{12}^{i} ; U_{21}^{j}$ & $U_{13}^{i} ; U_{31}^{j}$ \\
thầu j & $S_{2}^{j}$ & $U_{12}^{i} ; U_{21}^{j}$ & $U_{22}^{i} ; U_{22}^{j}$ & $U_{23}^{i} ; U_{32}^{j}$ \\
& $S_{3}^{j}$ & $U_{13}^{i} ; U_{31}^{j}$ & $U_{23}^{i} ; U_{32}^{j}$ & $U_{33}^{i} ; U_{33}^{j}$ \\
\hline
\end{tabular}

Xác suất mỗi tình huống là $\operatorname{prob}\left(B_{\alpha}^{i}, B_{\beta}^{j}\right), \forall \alpha, \beta \in n=\{1,2, \ldots, N\}$. Xác suất tổng xảy ra mỗi tình huống đấu thầu sẽ có dạng: $\operatorname{prob}($ case $)=\operatorname{prob}\left(C^{i}<C^{j}\right) \cap \operatorname{prob}\left[C^{i}<C^{j}<C^{i}+(n-1) \Delta\right]$, $\cap \operatorname{prob}\left(B_{\alpha}^{i} ; B_{\beta}^{j}\right), \forall \alpha, \beta \notin n=\{1,2,3 \ldots N\}$

được minh họa như Hình 5 .

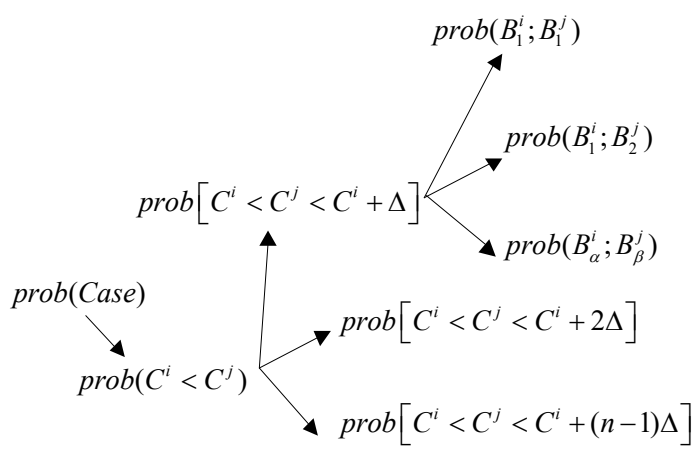

Hình 5. Sơ đồ cây xác suất các tình huống tương tác đấu thầu.

Việc nhận định thắng/thua trong mỗi tình huống được thực hiện bằng cách đối chiếu các giá trị giá gốc, giá mark-up,... Với phương pháp giá thấp nhất, nhà thầu có giá dự thầu thấp hơn sẽ thắng thầu, được thể hiện như Hình 6.

\section{SO SÁNH CÁC PHƯơNG ÁN CHIẾN THUẬT CỦA 2 NHÀ THẦU}

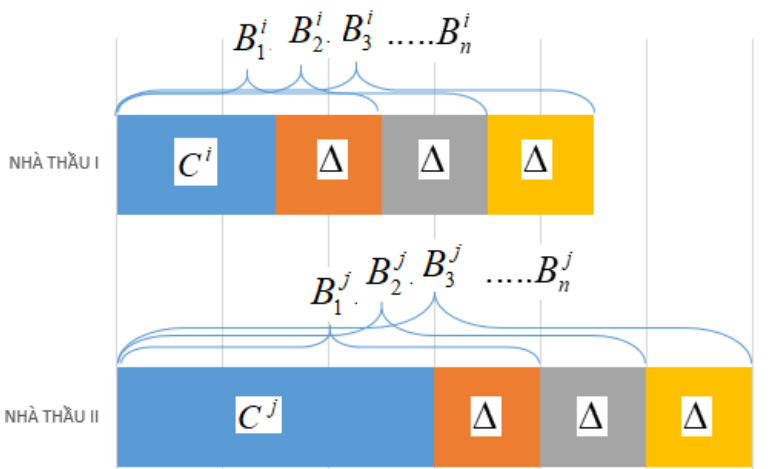

Hình 6. Sơ đồ so sánh các phương án chiến thuật.

\section{Trường hợp nghiên cứu}

Trong nghiên cứu, tác giả đã giả định một tình huống đấu thầu của nhà thầu $\mathrm{A}$ và hai nhà thầu đối thủ I và II (gọi tắt là nhà thầu I, nhà thầu II).

5.1. Các dữ liệu đầu vào 
Mức lợi nhuận cơ bản $\Delta=10000$ (ĐVT: Triệu đồng), Bảng 6 minh họa giá thầu cho ba phương án của nhà thầu $\mathrm{A}$, nhà thầu I và nhà thầu $\mathrm{II}$ gồm: Lời ít $\left(S_{1}^{i}\right)$, lời vừa $\left(S_{2}^{i}\right)$ và lời nhiều $\left(S_{3}^{i}\right)$; cụ thể như sau:

$$
\begin{aligned}
& S_{1}^{i}: B_{1}^{i}=C_{i}+\Delta \\
& S_{2}^{i}: B_{2}^{i}=C_{i}+2 \Delta \\
& S_{3}^{i}: B_{3}^{i}=C_{i}+3 \Delta \\
& i=A, I, I I
\end{aligned}
$$

\begin{tabular}{|c|c|c|c|c|c|}
\hline \multirow[b]{2}{*}{ Biến } & \multirow[b]{2}{*}{ Thang đo mức độ } & \multicolumn{3}{|c|}{ Xác suất } & \multirow{2}{*}{$\begin{array}{c}\text { Xu } \\
\text { hướng } \\
\text { khảo sát }\end{array}$} \\
\hline & & $\begin{array}{c}\text { Nhà } \\
\text { thầu A }\end{array}$ & $\begin{array}{l}\text { Nhà } \\
\text { thầu I }\end{array}$ & $\begin{array}{l}\text { Nhà } \\
\text { thầu II }\end{array}$ & \\
\hline \multicolumn{6}{|c|}{ Biến trung gian $\mathrm{A}$} \\
\hline \multirow{3}{*}{ A. 1} & Mức độ 1 & 0,42 & 0,36 & 0,85 & $S_{3}$ \\
\hline & Mức độ 2 & 0,35 & 0,42 & 0,15 & $S_{2}, S_{3}$ \\
\hline & Mức độ 3 & 0,23 & 0,22 & 0 & $S_{1}$ \\
\hline \multirow{3}{*}{ A. 2} & Mức độ 1 & 0,32 & 0,16 & 0,76 & $S_{1}$ \\
\hline & Mức độ 2 & 0,34 & 0,42 & 0,21 & $S_{2}$ \\
\hline & Mức độ 3 & 0,24 & 0,42 & 0,03 & \\
\hline \multirow{3}{*}{ A. 3} & Mức độ 1 & 0,57 & 0,26 & 0,56 & $S_{1}$ \\
\hline & Mức độ 2 & 0,21 & 0,49 & 0,21 & $S_{2}, S_{3}$ \\
\hline & Mức độ 3 & 0,22 & 0,25 & 0,23 & $S_{3}$ \\
\hline \multicolumn{6}{|c|}{ Biến trung gian $B$} \\
\hline \multirow{3}{*}{ B. 1} & Mức độ 1 & 0,32 & 0,32 & 0,32 & $S_{3}$ \\
\hline & Mức độ 2 & 0,43 & 0,43 & 0,43 & $S_{2}$ \\
\hline & Mức độ 3 & 0,25 & 0,25 & 0,25 & $S_{1}$ \\
\hline \multirow{3}{*}{ B. 2} & Mức độ 1 & 0,31 & 0,31 & 0,31 & $S_{3}$ \\
\hline & Mức độ 2 & 0,23 & 0,23 & 0,23 & $S_{2}$ \\
\hline & Mức độ 3 & 0,56 & 0,56 & 0,56 & $S_{1}$ \\
\hline \multirow{3}{*}{ B. 3} & Mức độ 1 & 0,4 & 0,4 & 0,4 & $S_{1}$ \\
\hline & Mức độ 2 & 0,2 & 0,2 & 0,2 & $S_{2}$ \\
\hline & Mức độ 3 & 0,4 & 0,4 & 0,4 & $S_{3}$ \\
\hline \multirow{3}{*}{ B. 4} & Mức độ 1 & 0,24 & 0,42 & 0,03 & $S_{3}$ \\
\hline & Mức độ 2 & 0,57 & 0,26 & 0,56 & $S_{2}$ \\
\hline & Mức độ 3 & 0,21 & 0,49 & 0,21 & \\
\hline B. 5 & Mức độ 1 & 0,2 & 0,2 & 0,2 & $S_{2}, S_{3}$ \\
\hline
\end{tabular}

\begin{tabular}{|c|c|c|c|c|c|}
\hline \multirow{4}{*}{ Biến } & \multirow[b]{2}{*}{ Thang đo mức độ } & \multicolumn{3}{|c|}{ Xác suất } & \multirow{2}{*}{$\begin{array}{c}\text { Xu } \\
\text { hướng } \\
\text { khảo sát }\end{array}$} \\
\hline & & $\begin{array}{c}\text { Nhà } \\
\text { thầu A }\end{array}$ & $\begin{array}{l}\text { Nhà } \\
\text { thầu I }\end{array}$ & $\begin{array}{l}\text { Nhà } \\
\text { thầu II }\end{array}$ & \\
\hline & Mức độ 2 & 0,23 & 0,23 & 0,23 & $S_{2}$ \\
\hline & Mức độ 3 & 0,57 & 0,57 & 0,57 & $S_{1}$ \\
\hline \multicolumn{6}{|c|}{ Biến trung gian Ca } \\
\hline \multirow{3}{*}{ C. 1} & Mức độ 1 & 0,23 & 0,56 & 0,56 & $S_{1}$ \\
\hline & Mức độ 2 & 0,3 & 0,21 & 0,22 & $S_{2}$ \\
\hline & Mức độ 3 & 0,47 & 0,23 & 0,22 & $S_{3}$ \\
\hline \multirow{3}{*}{ C. 2} & Mức độ 1 & 0,2 & 0,86 & 0,21 & $S_{1}$ \\
\hline & Mức độ 2 & 0,3 & 0,12 & 0,65 & $S_{2}$ \\
\hline & Mức độ 3 & 0,5 & 0,02 & 0,14 & $S_{3}$ \\
\hline \multirow{3}{*}{ C. 3} & Mức độ 1 & 0,4 & 0,26 & 0,15 & $S_{3}$ \\
\hline & Mức độ 2 & 0,3 & 0,15 & 0,62 & $S_{2}$ \\
\hline & Mức độ 3 & 0,3 & 0,59 & 0,23 & $S_{1}$ \\
\hline \multirow{3}{*}{ C. 4} & Mức độ 1 & 0,21 & 0,26 & 0,69 & $S_{1}$ \\
\hline & Mức độ 2 & 0,26 & 0,15 & 0,14 & $S_{2}$ \\
\hline & Mức độ 3 & 0,53 & 0,59 & 0,27 & $S_{3}$ \\
\hline \multicolumn{6}{|c|}{ Biến trung gian $\mathrm{Cb}$} \\
\hline \multirow{3}{*}{ C. 5} & Mức độ 1 & 0,8 & 0,75 & 0,12 & $S_{1}$ \\
\hline & Mức độ 2 & 0,1 & 0,15 & 0,25 & $S_{2}$ \\
\hline & Mức độ 3 & 0,1 & 0,1 & 0,63 & $S_{3}$ \\
\hline \multirow{3}{*}{ C. 6} & Mức độ 1 & 0,6 & 0,1 & 0,5 & $S_{1}$ \\
\hline & Mức độ 2 & 0,2 & 0,6 & 0,25 & \\
\hline & Mức độ 3 & 0,2 & 0,3 & 0,25 & \\
\hline \multirow{3}{*}{ C.7 } & Mức độ 1 & 0,4 & 0,26 & 0,16 & $S_{3}$ \\
\hline & Mức độ 2 & 0,3 & 0,56 & 0,36 & $S_{1} S_{2}$ \\
\hline & Mức độ 3 & 0,3 & 0,18 & 0,58 & \\
\hline \multirow{3}{*}{ C. 8} & Mức độ 1 & 0,7 & 0,5 & 0,32 & $S_{1}$ \\
\hline & Mức độ 2 & 0,2 & 0,2 & 0,12 & $S_{1}, S_{2}$ \\
\hline & Mức độ 3 & 0,1 & 0,3 & 0,56 & $S_{3}$ \\
\hline \multirow{3}{*}{ C. 9} & Mức độ 1 & 0,6 & 0,7 & 0,5 & $S_{3}$ \\
\hline & Mức độ 2 & 0,4 & 0,3 & 0,5 & $S_{2}$ \\
\hline & Mức độ 3 & 0 & 0 & 0 & \\
\hline
\end{tabular}

Bảng 6.

Phân bố xác suất ba mức độ của 28 nhân tố. 


\begin{tabular}{|c|c|c|c|c|c|}
\hline \multirow[b]{2}{*}{ Biến } & \multirow[b]{2}{*}{ Thang đo mức độ } & \multicolumn{3}{|c|}{ Xác suất } & \multirow{2}{*}{$\begin{array}{c}\text { Xu } \\
\text { hướng } \\
\text { khảo sát }\end{array}$} \\
\hline & & $\begin{array}{c}\text { Nhà } \\
\text { thầu A }\end{array}$ & $\begin{array}{c}\text { Nhà } \\
\text { thầu I }\end{array}$ & $\begin{array}{c}\text { Nhà } \\
\text { thầu II }\end{array}$ & \\
\hline \multirow{3}{*}{ C. 10} & Mức độ 1 & 0,5 & 0,4 & 0,8 & $S_{1}$ \\
\hline & Mức độ 2 & 0,5 & 0,6 & 0,2 & $S_{1}$ \\
\hline & Mức độ 3 & 0 & 0 & 0 & \\
\hline \multirow{3}{*}{ C.11 } & Mức độ 1 & 0,31 & 0,31 & 0,31 & $S_{1}$ \\
\hline & Mức độ 2 & 0,23 & 0,23 & 0,23 & $S_{2}$ \\
\hline & Mức độ 3 & 0,56 & 0,56 & 0,56 & $S_{3}$ \\
\hline \multirow{3}{*}{ C.12 } & Mức độ 1 & 0,4 & 0,4 & 0,4 & $S_{1}$ \\
\hline & Mức độ 2 & 0,2 & 0,2 & 0,2 & $S_{2}$ \\
\hline & Mức độ 3 & 0,4 & 0,4 & 0,4 & $S_{3}$ \\
\hline \multicolumn{6}{|c|}{ Biến trung gian $\mathrm{Cc}$} \\
\hline \multirow{3}{*}{ C. 13} & Mức độ 1 & 0,6 & 0,7 & 0,5 & $S_{3}$ \\
\hline & Mức độ 2 & 0,4 & 0,3 & 0,5 & $S_{1}, S_{2}$ \\
\hline & Mức độ 3 & 0 & 0 & 0 & \\
\hline \multirow{3}{*}{ C.14 } & Mức độ 1 & 0,5 & 0,4 & 0,8 & $S_{3}$ \\
\hline & Mức độ 2 & 0,5 & 0,6 & 0,2 & $S_{1}, S_{2}$ \\
\hline & Mức độ 3 & 0 & 0 & 0 & \\
\hline \multirow{3}{*}{ C.15 } & Mức độ 1 & 0,9 & 0,6 & 0,3 & $S_{3}$ \\
\hline & Mức độ 2 & 0,1 & 0,4 & 0,7 & $S_{1}, S_{2}$ \\
\hline & Mức độ 3 & 0 & 0 & 0 & \\
\hline \multicolumn{6}{|c|}{ Biến trung gian $D$} \\
\hline \multirow{3}{*}{ D. 1} & Mức độ 1 & 0,4 & 0,4 & 0,4 & $S_{1}$ \\
\hline & Mức độ 2 & 0,3 & 0,3 & 0,3 & \\
\hline & Mức độ 3 & 0,3 & 0,3 & 0,3 & $S_{3}$ \\
\hline \multirow{3}{*}{ D. 2} & Mức độ 1 & 0,3 & 0,12 & 0,65 & \\
\hline & Mức độ 2 & 0,5 & 0,02 & 0,14 & \\
\hline & Mức độ 3 & 0,4 & 0,26 & 0,15 & \\
\hline \multirow{3}{*}{ D. 3} & Mức độ 1 & 0,6 & 0,6 & 0,6 & $S_{3}$ \\
\hline & Mức độ 2 & 0,2 & 0,2 & 0,2 & \\
\hline & Mức độ 3 & 0,2 & 0,2 & 0,2 & $S_{1}$ \\
\hline
\end{tabular}

Đối với Biến trung gian, tác giả chọ hệ số $\alpha=0,5$ cho tất cả các trường hợp.
Mối quan hệ nguyên nhân - kết quả được thể hiện theo Bảng 7, trong giới hạn của nghiên cứu, tác giả lấy hệ số $\mathrm{K}=1$. Việc đánh giá mối quan hệ trong từng tổ hợp được tác giả chia đều cho các khả năng xảy ra. Tức là $\beta=0,5$ đối với tổ hợp thuộc nhóm $2, \beta=0,33$ đối với tổ hợp thuộc nhóm 3.

Bảng 7. Xác định mối quan hệ nguyên nhân - kết quả giữa các biến.

\begin{tabular}{clc}
\hline STT & Nhân tố nguyên nhân & Nhân tố kết quả \\
\hline 1 & B1, B3, B4, B5 & B2 \\
\hline 2 & B5 & C1 \\
\hline 3 & C7, C9 & C8 \\
\hline 4 & B2, B5, C9, C12 & C11 \\
\hline
\end{tabular}

Phân bố xác suất giá gốc cuả ba nhà thầu:

+ Nhà thầu $\mathrm{A}$ - Nhà thầu $\mathrm{I}$ :

Giá gốc: $C^{A}>C^{I}$

Xác suất tương quan giá gốc với các phương án giá nhà thầu I: $\operatorname{prob}\left(C^{I}<C^{A}<B_{1}^{I}\right)=0,3 ; \operatorname{prob}\left(C^{I}<B_{1}^{I}<C^{A}<B_{2}^{I}\right)=0,2$

$\operatorname{prob}\left(C^{I}<B_{2}^{I}<C^{A}<B_{3}^{I}\right)=0,5$

+ Nhà thầu $\mathrm{A}$ - Nhà thầu II:

Giá gốc: $C^{A}<C^{I I}$

Xác suất tương quan giá gốc với các phương án giá nhà thầu II: $\operatorname{prob}\left(C^{A}<C^{I I}<B_{1}^{I I}\right)=0,5$

$\operatorname{prob}\left(C^{A}<B_{1}^{A}<C^{I}<B_{2}^{A}\right)=0,4$

$\operatorname{prob}\left(C^{A}<B_{2}^{A}<C^{I I}<B_{3}^{A}\right)=0,1$

Về đánh giá các mối quan hệ nguyên nhân - kết quả, giả sử khả năng các mối quan hệ ảnh hưởng như nhau, và xác suất xảy ra chia đều cho các trạng thái mức độ.

\subsection{Kết quả}

Bảng 8.

Tổng hợp kết quả xác suất lựa chọn các chiến thuật của nhà thầu A.

\begin{tabular}{cccc}
\hline STT & Tên chiến thuật & Kết quả từ mô hình & Xác suất lựa chọn \\
\hline 1 & $S_{1}^{A}$ & 0,401 & $33,56 \%$ \\
\hline 2 & $S_{2}^{A}$ & 0,387 & $32,38 \%$ \\
\hline 3 & $S_{3}^{A}$ & 0,407 & $34,06 \%$
\end{tabular}

\section{Bảng 9.}

Tổng hợp kết quả xác suất lựa chọn các chiến thuật của nhà thầu đối thủ I.

STT Tên chiến thuật Kết quả từ mô hình Xác suất lựa chọn

\begin{tabular}{cccc}
\hline 1 & $S_{1}^{I}$ & 0,386 & $31 \%$ \\
\hline 2 & $S_{2}^{I}$ & 0,456 & $36,63 \%$ \\
\hline 3 & $S_{3}^{I}$ & 0,403 & $32,37 \%$ \\
\hline
\end{tabular}


Bảng 10.

Tổng hợp kết quả xác suất lựa chọn các chiến thuật của nhà thầu đối thủ II.

\begin{tabular}{cccc}
\hline STT & Tên chiến thuật & $\begin{array}{c}\text { Kết quả từ mô } \\
\text { hình }\end{array}$ & Xác suất lựa chọn \\
\hline 1 & $S_{1}^{I I}$ & 0,432 & $34,53 \%$ \\
\hline 2 & $S_{2}^{I I}$ & 0,476 & $38,05 \%$ \\
\hline 3 & $S_{3}^{I I}$ & 0,343 & $27,42 \%$ \\
\hline
\end{tabular}

* Đánh giá kết quả đầu ra của mô hình xác định xác suất các chiến thuật:

Kết quả đầu ra thể hiện xác suất lựa chọn từng chiến thuật của các nhà thầu. Tuy nhiên, tổng kết quả đầu ra của ba chiến thuật không thể bằng 1 do xu hướng lựa chọn chiến thuật không bao phủ toàn bộ các nhân tố, có trường hợp trùng lặp, mối quan hệ nguyên nhân - kết quả đan xen. Vì vậy, để tiện theo dõi, tác giả đã quy ra xác suất lựa chọn bằng phương pháp tỷ lệ, được trình bày trong các Bảng 8, Bảng 9 và Bảng 10.

Bảng 11.

Kết quả sau đánh giá tương tác giữa nhà thầu $\mathrm{A}$ và nhà thầu đối thủ $\mathrm{I}$.

\begin{tabular}{|c|c|c|c|c|}
\hline \multirow{2}{*}{ Nhà thầu } & \multicolumn{2}{|c|}{ Phương án hợp lý } & \multicolumn{2}{c|}{$\begin{array}{c}\text { Phương án có xác suất xảy } \\
\text { ra thắng thầu lớn nhất }\end{array}$} \\
\cline { 2 - 5 } & Lợi nhuận & $\begin{array}{c}\text { Kết quả từ } \\
\text { mô hình }\end{array}$ & Lợi nhuận & $\begin{array}{c}\text { Kết quả từ } \\
\text { mô hình }\end{array}$ \\
\hline A & 20000 & 0,046 & 10000 & 0,054 \\
\hline I & 30000 & 0,082 & 20000 & 0,092 \\
\hline
\end{tabular}

\section{Bảng 12.}

Kết quả sau đánh giá tương tác giữa nhà thầu $\mathrm{A}$ và nhà thầu đối thủ II.

\begin{tabular}{|c|c|c|c|c|}
\hline \multirow{2}{*}{ Nhà thầu } & \multicolumn{2}{|c|}{ Phương án hợp lý } & \multicolumn{2}{|c|}{$\begin{array}{c}\text { Phương án có xác suất xảy } \\
\text { ra thắng thầu lớn nhất }\end{array}$} \\
\cline { 2 - 5 } & Lợi nhuận & $\begin{array}{c}\text { Kết quả từ } \\
\text { mô hình }\end{array}$ & Lợi nhuận & $\begin{array}{c}\text { Kết quả từ } \\
\text { mô hình }\end{array}$ \\
\hline A & 30000 & 0,077 & 10000 & 0,095 \\
\hline II & 20000 & 0,096 & 20000 & 0,096 \\
\hline
\end{tabular}

\section{* Đánh giá kết quả đầu ra của mô hình đánh giá tương tác giữa} nhà thầu $\mathrm{A}$ và nhà thầu $\mathrm{I}, \mathrm{II}$ :

Kết quả từ mô hình là số liệu thể hiện khả năng xảy ra của phương án lợi nhuận hợp lý và phương án có xác suất thắng thầu lớn nhất của nhà thầu $\mathrm{A}$ với từng nhà thầu I và II (Bảng 11, Bảng 12). Nhà thầu $\mathrm{A}$ sẽ dựa vào mức lợi nhuận và mức chênh lệch xác suất thắng thầu của hai phương án (hợp lý, khả năng thắng thầu lớn nhất) để ra quyết định lựa chọn giải pháp đấu thầu cho mình. Kết quả từ mô hình phương án có xác suất thắng thầu bé, điều này không có nghĩa là khả năng thắng thầu thấp. Vì để xác định được các giá trị này qua quá trình phân chia, cần đánh giá nhiều trường hợp, và mô hình này không cung cấp độ lớn của các phương án nhà thầu có tham gia dự thầu hay không, mà cung cấp cơ sở để nhà thầu tham gia dự thầu.

\section{Kết luận}

Nghiên cứu này cung cấp một mô hình sử dụng mạng BBNs và lý thuyết trò chơi để xác định xác suất thắng thầu, để nhà thầu có cơ sở ra quyết định giá dự thầu. Tuy nhiên, để vận dụng được mô hình này, nhà thầu phải rất am hiểu về lĩnh vực xây dựng nhằm đưa ra các nhận định về các đặc điểm bản thân, nhà thầu đối thủ, dự án tham gia,... cũng như xu hướng lựa chọn chiến thuật. Hạn chế của mô hình là dữ liệu đầu vào nhiều, quá trình tính toán trải qua nhiều bước, nhiều giai đoạn khiến cho dễ xảy ra sai sót. Vì vậy, để có thể thực hiện trên nhiều chiến thuật hơn, mô hình nên được hoàn thiện cùng với các giải pháp phần mềm và công nghệ tiên tiến.

\section{Tài liệu tham khảo}

[1]. M. Jarkas Abdulaziz, A. Mubarak Saleh, and Y. Kadri Charles, "Critical Factors Determining Bid/No Bid Decisions of Contractors in Qatar," Journal of Management in Engineering, vol. 30, no. 4, p. 05014007, 2014/07/01 2014, doi: 10.1061/(ASCE)ME.1943-5479.0000223.

[2]. A. A. Shash, "Factors considered in tendering decisions by top UK contractors," Construction Management and Economics, vol. 11, no. 2, pp. 111-118, 1993/03/01 1993, doi: 10.1080/01446199300000004.

[3]. I. Ahmad and I. Minkarah, "Questionnaire Survey on Bidding in Construction," Journal of Management in Engineering, vol. 4, no. 3, pp. 229243, 1988/07/01 1988, doi: 10.1061/(ASCE)9742-597X(1988)4:3(229).

[4]. Y.-T. C. Ching-Torng Lin, "Bid/no-bid decision-making - a fuzzy linguistic approach," International Journal of Project Management, vol. 22, pp. 585593, 2004.

[5]. C. I. Dong-hong, Xi-yan, Zhang2, "APPLICATION OF GAME THEORY ON BIDDING PRICE DECISION," 2009.

[6]. T. Hegazy, Computer-Based Construction Project Management. 2002. 